\title{
Design-in Reliability for Modern Wavelength-division Multiplex (WDM) Distributed Feedback (DFB) InP Lasers
}

\author{
Jia-Sheng Huang ${ }^{1}$ \\ ${ }^{1}$ Emcore, Broadband Division, 2015 W. Chestnut Street, Alhambra, CA 91803, USA \\ Correspondence: Jia Sheng Huang, Emcore, Broadband Division, 2015 W. Chestnut Street, Alhambra, CA \\ 91803. Tel: 1-626-293-3622. E-mail: jshuang@emcore.com
}

Received: February 26, 2012

Accepted: March 12, $2012 \quad$ Online Published: May 1, 2012

doi:10.5539/apr.v4n2p15

URL: http://dx.doi.org/10.5539/apr.v4n2p15

\begin{abstract}
Due to the increasing channel density in WDM network, the reliability requirement of each channel has become increasingly stringent. To meet the stringent WDM reliability requirement, it is necessary to achieve stable threshold current and wavelength over time. In this paper, we study the design-in reliability of DFB InP lasers involving the controlling parameters such as p-metal contact, epitaxial regrowth interface, substrate quality and laser cavity length. We will discuss the influence of each parameter and physical mechanism. We demonstrate significant reliability improvement with incorporation of design and process optimization. We also discuss the correlation of reliability degradation and wavelength drift.
\end{abstract}

Keywords: semiconductor lasers, reliability, wavelength division multiplexing, semiconductor heterojunctions, semiconductor devices, optoelectronics

\section{Introduction}

The modern WDM network requires increasing number of channels in order to meet broadband applications. Due to the increasing channel density, the reliability of each channel has become more stringent than ever. The reliability requirement expected by the end-users or network operators typically consists of two aspects: wavelength stability and threshold current robustness. While these two aspects are seemingly two different specifications, they are essentially correlated. For example, as the threshold current increases during aging, it requires higher operating bias current to maintain the same level of optical power. The increase in the operating current consequently results in higher junction temperature and longer wavelength. In the past, most of the wavelength stability requires $<=0.1 \mathrm{~nm}$ for $100 \mathrm{G}$ DWDM spacing. Recently, many network and/or cable operators request the laser diode component manufacturers to meet wavelength stability of $0.03-0.09 \mathrm{~nm}$ (Huang, 2011).

Etched mesa buried heterostructure $(\mathrm{BH}) \mathrm{InGaAsP} / \mathrm{InP}$ semiconductor laser diodes are widely used in 1.3-1.5micrometer wavelength photonic products due to their excellent characteristics of low threshold and high power (Itoh et al., 1966; Mizuishi et al., 1983; Ishino et al., 1997; Fukuda et al., 1987). However, the BH lasers tend to show more reliability degradation than the ridge waveguide (RWG) lasers (Huang, 2005). The latter is often characterized with random failures, while the former tends to show gradual degradation in a more predictable manner.

In this study, we demonstrate design-in reliability of BH lasers by controlling parameters such as p-metal contact, epitaxial regrowth interface, substrate quality and laser cavity length. We have achieved significant reliability improvement by optimizing each parameter. We demonstrate that the $\mathrm{BH}$ lasers could meet the stringent reliability requirement for modern WDM network.

\section{Experimental}

Edge-emitting distributed feedback (DFB) lasers with O-band (1310 nm and vicinity) and C-band (1550 nm and vicinity) lasing wavelengths were fabricated. Figure 1 shows the schematic of the DFB buried heterostructure (BH) laser. Epitaxial layers were grown on Sn-doped or S-doped n-type InP substrates using metal organic chemical vapor deposition (MOCVD) technique. First, n-doped InP buffer layer of 0.5 micrometer or 1.4 micrometer thickness was grown. An active layer consisting of multi-quantum well structures and separate confinement heterostructure ( $\mathrm{SCH}$ ) were grown on top of the buffer. The grating layers were grown sequentially. 
The grating contrast was formed by lithography and wet etch using holographic technique. The composition of the active region was InGaAsP.

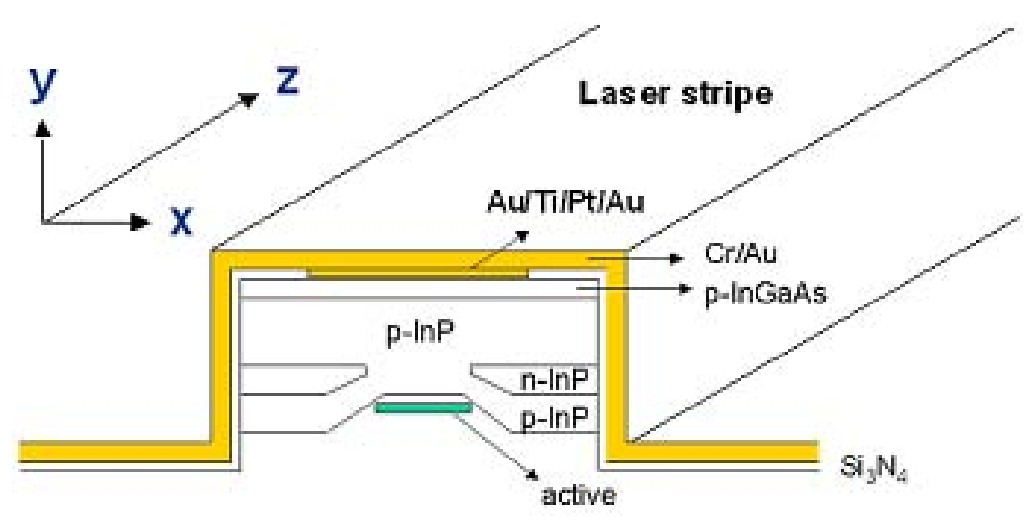

$n-\ln P$

\section{Au/Ge/NirAu}

Figure 1. Schematic of DFB buried heterostructure laser

A mesa structure was formed by wet etch. Subsequently, $\mathrm{p}$-InP and $\mathrm{n}$-InP burying layers were grown to form current blocking. The wafer was then etched into mesa structures covered with dielectric layers. The contact opening on the dielectric was created by the reactive ion etching (RIE), and the p-metal stack was deposited to make ohmic contact. The p-metal stack consisted of $\mathrm{Au} / \mathrm{Ti} / \mathrm{Pt} / \mathrm{Au}$ on the p-contact area and $\mathrm{Cr} / \mathrm{Au}$ metallization on the bondpad area.

Finally, wafers were cleaved into bars to form facets coated with anti-reflective (AR) and highly-reflective (HR) on the front and rear facets, respectively. Chips were die attached to the AIN submounts to facilitate light vs. current (LI) testing. The chip-on-submounts were screened by burn-in, and the chips that passed the burn-in screening were used for life tests.

To study the defects, the laser samples were prepared by focused ion beam using life-off technique (Giannuzzi \& Stevie, 1999; Huang et al., JMR 2000; Huang et al., JAP 2000). Scanning electron microscopy (SEM), transmission electron microscopy (TEM) and scanning transmission electron microscopy (STEM) were utilized. The STEM was also equipped with energy dispersive spectroscopy by X-ray (EDS). The samples were analyzed by Evans Analytical Group (Raleigh, NC).

\section{Results and Discussions}

Long-term device reliability is typically characterized by the increase in threshold current (Ith) and the decrease in optical output power (Huang, 2005). The laser degradation associated with the changes in the threshold current and optical power or overall drop in lasing efficiency are a result of defect formation in the active region. The reliability of laser devices is often determined by the defects in the active region such as the dark-spot defect (DSD) and dark-line defect (DLD) (Kallstenius et al., 1999; Fukuda et al., 1983; Chin et al., 1984).

For C-band $1550 \mathrm{~nm}$ lasers of 550 micrometer cavity length, the typical threshold current and output power are in the ranges of 12-15 mA and 38-48 mW, respectively. After 24-hour burn-in, the threshold current and output power increase to $16-19 \mathrm{~mA}$ and $35-45 \mathrm{~mW}$, respectively. Figure 2 shows an example of LI characteristics before and after burn-in stress. After the 24-hour burn-in, the lasers exhibited degradation in both threshold current and output power. Upon further burn-in stressing, the lasers stabilized with little change. In this case, the threshold current increased from 16.4 to $25.6 \mathrm{~mA}$, and the output power decreased from 37.0 to $30.3 \mathrm{~mW}$.

In the following, we will present experimental data and discuss the determining factors that are responsible for laser degradation and the defect formation in the active. 


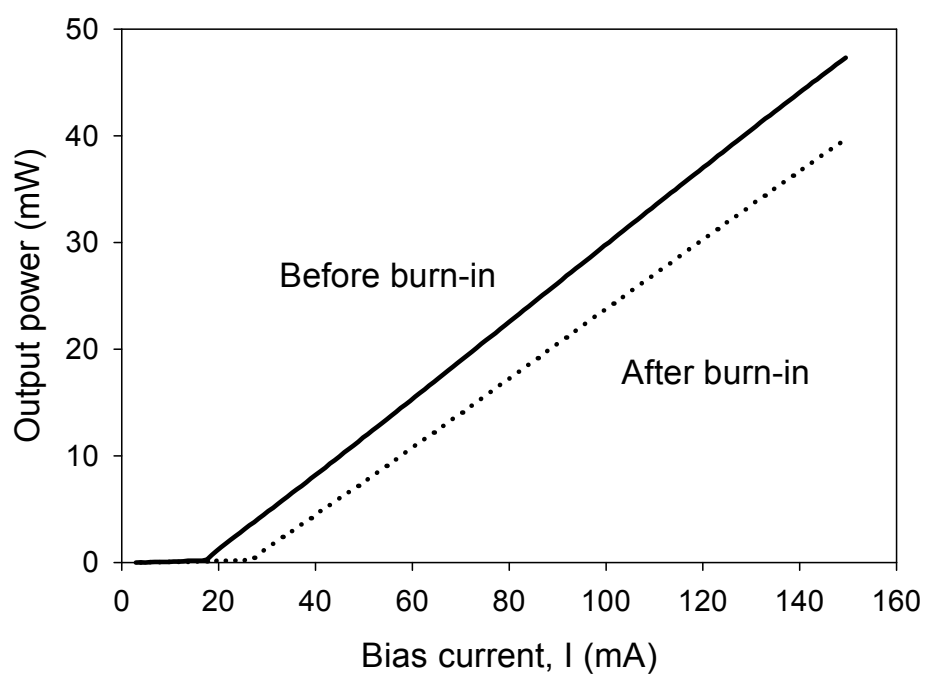

Figure 2. Example of light vs. current (LI) plot of a 1550nm DFB laser before and after 24-hour burn-in. The LI was taken at $25 \mathrm{C}$

\subsection{P-metal Contact}

The p-type metal contact and its interaction with heavily p-doped epitaxial contact layer are critical for device burn-in and reliability performances. The metal species and the underlying contact epi-layer typically determine the interfacial reaction. The stability of the interfacial metallic compounds has significant impact in long-term reliability.

When the interfacial metallic compounds are properly formed, a good Ohmic contact and resultant low laser resistance can be achieved while maintaining good long-term stability. However, the laser diode could degrade over time if there are excessive defects or Au diffusion in the p-metal contact. Those defects could propagate towards the active region during device operation or aging.

\subsubsection{Contact Etch}

One of the laser degradation mechanisms is associated with defect formation and propagation in the p-contact. Figure 3 shows the distributions of threshold current change of two experimental groups with different contact etch conditions. The lasers of both contact etch groups were subjected to the same aging condition with stress current of $175 \mathrm{~mA}$ at the case temperature of $100 \mathrm{C}$ for 48 hours. At the nominal device operating current, the laser junction temperature was about $30 \mathrm{C}$ above the case temperature (Huang et al., 2007). The lasers in one group were processed with $240 \mathrm{sec}$ of contact etch by reactive ion etching (RIE) while the lasers in the other were etched with $80 \mathrm{sec}$. The Ith change in the former was very small (around $0.8-1.2 \mathrm{~mA}$ in median). On the other hand, the Ith change in the $240 \mathrm{sec}$ RIE was $12 \mathrm{~mA}$, larger by a factor of 12 .

Since interfacial diffusion is driven by defects, the quality of the epitaxial contact layer is expected to influence the defect formation and propagation towards the active region. When the contact etch time is excessive, there are likely more defects generated by plasma charging damage during the dry etch. Those defects that originate from the p-contact could subsequently propagate towards the active region over time during aging. The defects could also enhance the Au diffusion at the p-contact.

The damaged region induced by RIE was likely a superficial layer that behaved as deep traps that captured conduction electrons. Foad et al proposed the conduction technique to estimate the extent of surface etching damage (Foad et al., 1993). Morello et al. reported that the damaged depth induced by RIE was about 21-50 nm measured by the conduction technique (Morello et al., 2006). The damage generation rate depended upon the energy of impinging ions.

The RF power, reactant, carrier gas flow and chamber pressure are also important for the contact etch in order to minimize the plasma damage (Pearton et al., 1992; Yang et al., 2007; Dylewicz et al., 2010; Cheung et al., 1994). 


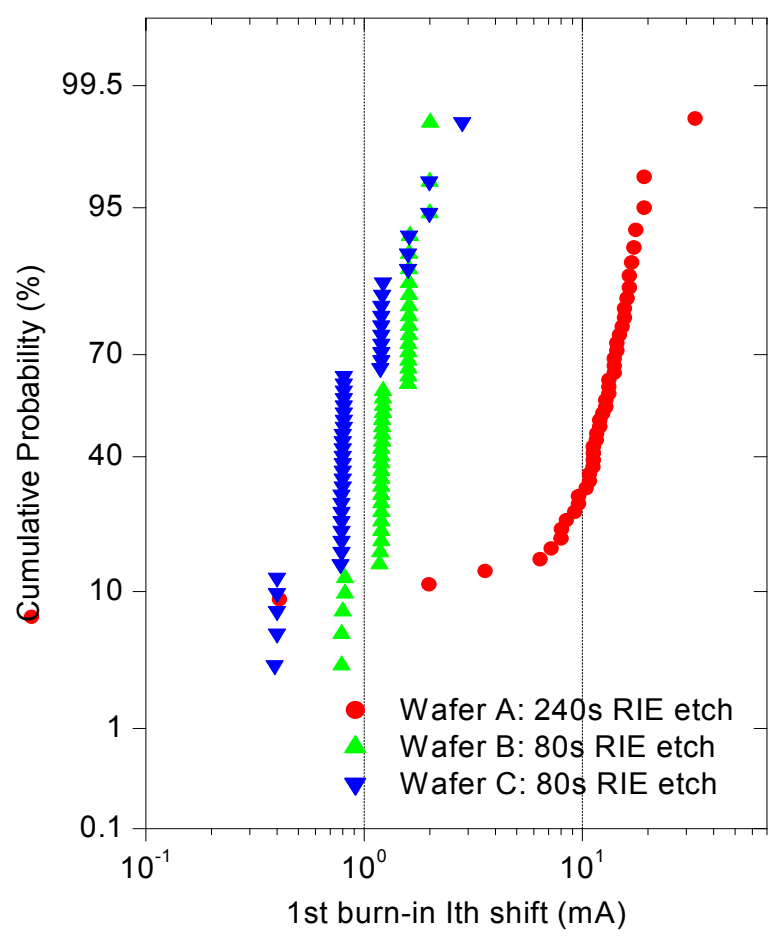

Figure 3. Distributions of threshold current (Ith) change of two contact etch groups. One group was etched with 240sec RIE while the other was etched with 80sec RIE

\subsection{2 p-InGaAs Contact Layer Thickness}

The contact layer thickness is also found to be influential in laser degradation. Figure 4 shows the Ith change as a function of contact layer thickness. Laser diodes of different InGaAs thickness ranging from 0.1 to 0.4 micrometer were compared. The contact layer thickness was measured by the scanning electron microscopy (SEM) and transmission electron microscopy (TEM). The Ith change of the 0.1 micrometer InGaAs lasers showed the largest Ith change, suggesting that the lasers with the 0.1 micrometer InGaAs were most vulnerable to laser degradation. In addition, the range of the Ith changes of the 0.1 micrometer InGaAs group was larger than the others, implying that the process window was less tolerant. As the InGaAs thickness increased, the Ith change become smaller, indicating that the lasers with the thicker InGaAs were more reliable.

As the InGaAs thickness decreased, it became easier for defects to propagate through the InGaAs layer into the InP. In addition, the thinner InGaAs also made the underlying InP more susceptible to the plasmas damage generated during the RIE etch. Finally, the Au diffusion in the $\mathrm{Au} / \mathrm{Ti} / \mathrm{Pt} / \mathrm{Au}$ to $\mathrm{p}+-\mathrm{InGaAs}$ contact was typically 0.1 micrometer in depth and confined to the InGaAs layer (Huang \& Vartuli, 2003). The Au diffusion into the InP became increasingly likely when the InGaAs thickness was 0.1-0.2 micrometer.

Figure 5 shows the TEM images of the defect punch-through in the thin InGaAs layer. The InGaAs layer thickness in this case was about 0.1 micrometer. The punch-through damaged area was likely due to the variation in the crystal composition resulting from interfacial diffusion. Figure 6 shows the STEM depth profile scan across the p-InGaAs and the underlying InP layer. The STEM scan shows the punch-through damage involved the interfacial diffusion among the chemical species of In, P, Ga and As. 
Ith change vs. p-InGaAs thickness

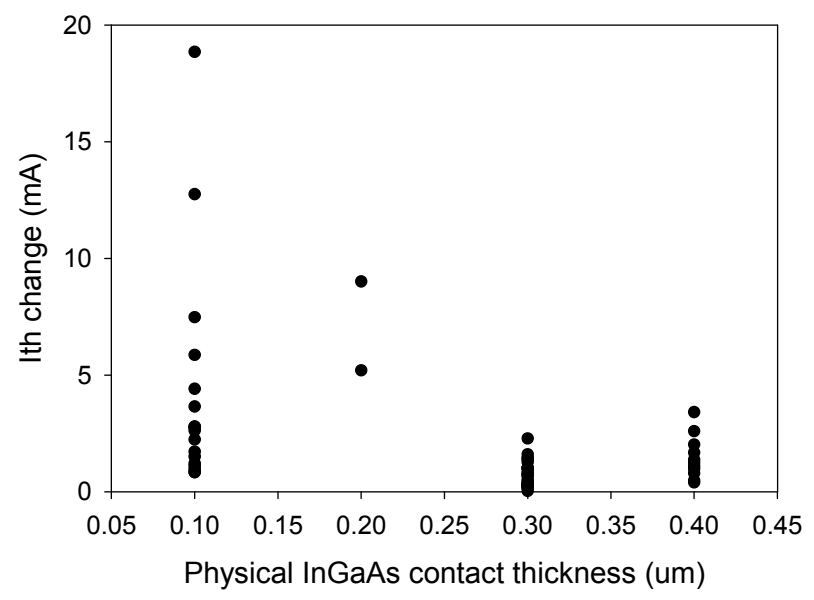

Figure 4. Threshold current (Ith) change as a function of InGaAs contact layer thickness. The lasers with thinner InGaAs contact layer tend to show larger Ith shift during aging

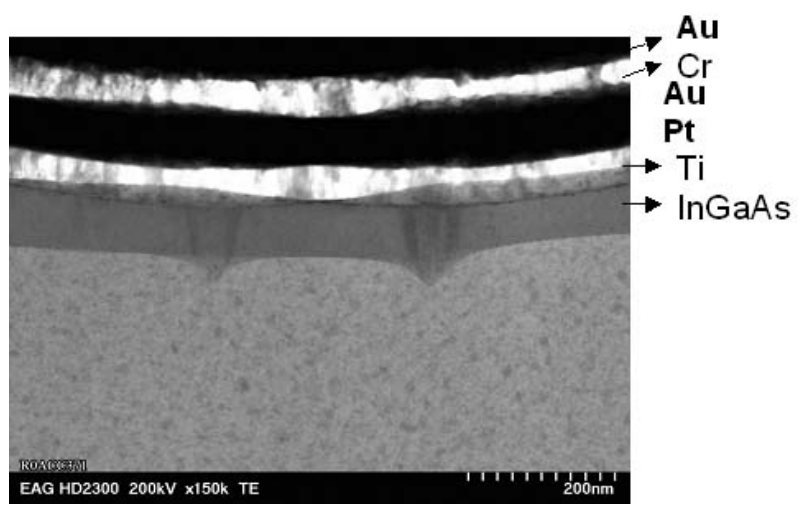

Figure 5. TEM bright field image of the p-contact to $\mathrm{p}$-InGaAs/InP showing the punch through related to interfacial diffusion

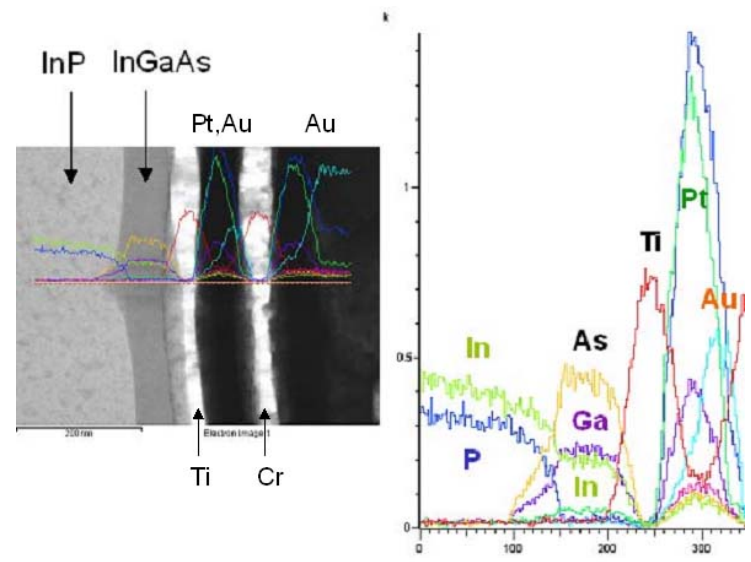

Figure 6. STEM depth profile scan of the p-contact to $\mathrm{p}$-InGaAs/InP showing the interfacial diffusion between InP and InGaAs 


\subsubsection{P-metallization}

It has been reported that $\mathrm{Au} / \mathrm{Zn}$ p-contact has adverse effect on laser degradation compared to $\mathrm{Au} / \mathrm{Ti} / \mathrm{Pt} / \mathrm{Au}$ contact (Huang \& Vartuli, 2004). The faster degradation in the $\mathrm{Au} / \mathrm{Zn}$ contact was attributed to defect formation resulting from the migration of $\mathrm{Au}$. Besides the faster diffusion rate, the alloy spike in the $\mathrm{Au} / \mathrm{Zn}$ contact could further accelerate the degradation due to the concentration of current flow in the low resistivity of the alloy compounds. Figure 7 shows the Ith and power change box plots of the lasers of the $\mathrm{Au} / \mathrm{Zn} / \mathrm{Cr} / \mathrm{Au}$ (thick) and $\mathrm{Au} / \mathrm{Ti} / \mathrm{Pt} / \mathrm{Au} / \mathrm{Cr} / \mathrm{Au}$ (thick) groups. The change in threshold current and output power of the $\mathrm{Au} / \mathrm{Ti} / \mathrm{Pt} / \mathrm{Au}$ were significantly less than those of the $\mathrm{Au} / \mathrm{Zn}$. The reliability improvement of the $\mathrm{Au} / \mathrm{Ti} / \mathrm{Pt} / \mathrm{Au}$ was attributed to the diffusion barrier by the Pt layer. As shown by the previous study (Huang \& Vartuli, 2004), the Pt barrier prevented the thick $\mathrm{Au}$ from interacting with the InGaAs and underlying InP layers, thus limiting the $\mathrm{Au}$ interfacial reaction. Figure $8(\mathrm{a})$ and (b) show the TEM images of the $\mathrm{Au} / \mathrm{Zn} / \mathrm{Cr} / \mathrm{Au}$ (thick) and $\mathrm{Au} / \mathrm{Ti} / \mathrm{Pt} / \mathrm{Au} / \mathrm{Cr} / \mathrm{Au}$ (thick) contacts, respectively. For the $\mathrm{Au} / \mathrm{Zn} / \mathrm{Au}$ samples, the Au-rich compounds penetrated deeper, consuming most or all the p-InGaAs layer. For the $\mathrm{Au} / \mathrm{Ti} / \mathrm{Pt} / \mathrm{Au} / \mathrm{Cr} / \mathrm{Au}$, the formation of the $\mathrm{Au}-\mathrm{Ga}$, Ti-As and Au-Ga-In metallic compounds was confined to the contact interface.
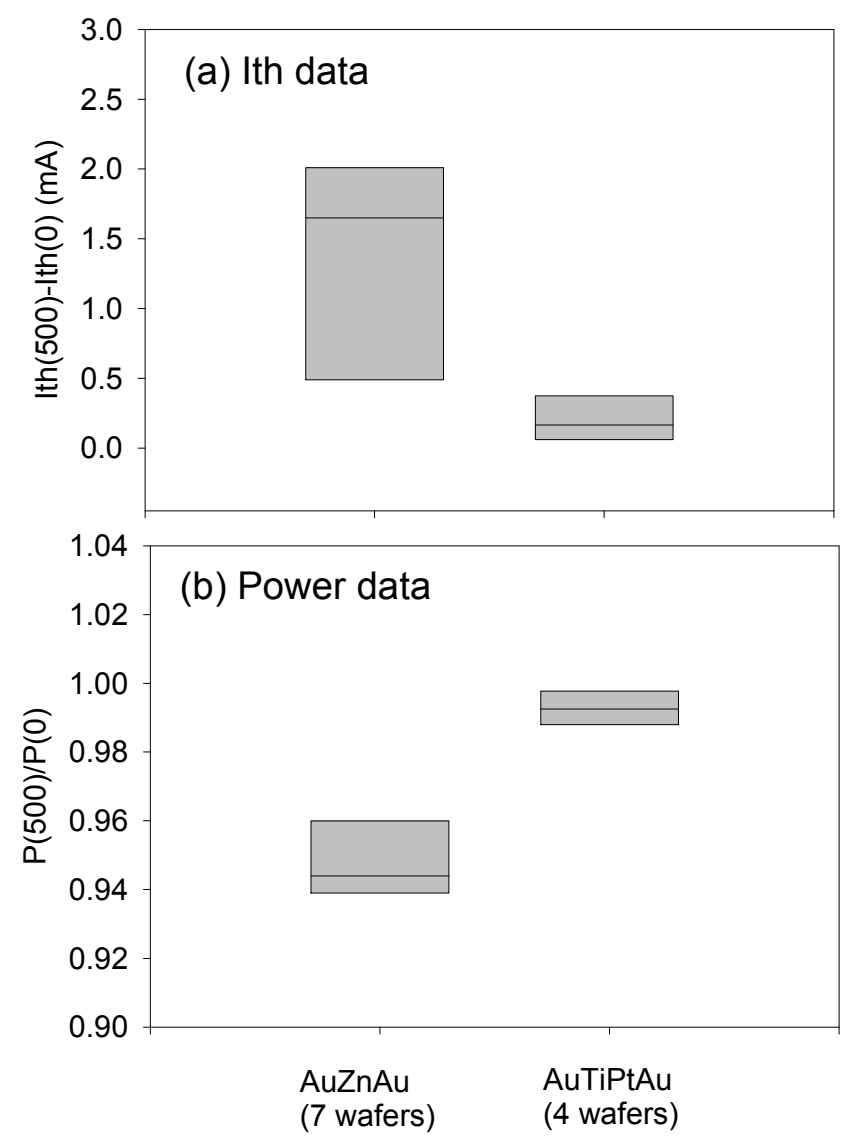

Figure 7. Box plots of the changes in (a) threshold current and (b) output power of the lasers of the $\mathrm{Au} / \mathrm{Zn} / \mathrm{Cr} / \mathrm{Au}$ and $\mathrm{Au} / \mathrm{Ti} / \mathrm{Pt} / \mathrm{Au} / \mathrm{Cr} / \mathrm{Au}$ groups. The Ith change is shown by the absolute change before and after 500 hours of stress aging, and the power change is the ratio of post-aging power after 500 hours vs. pre-aging power 

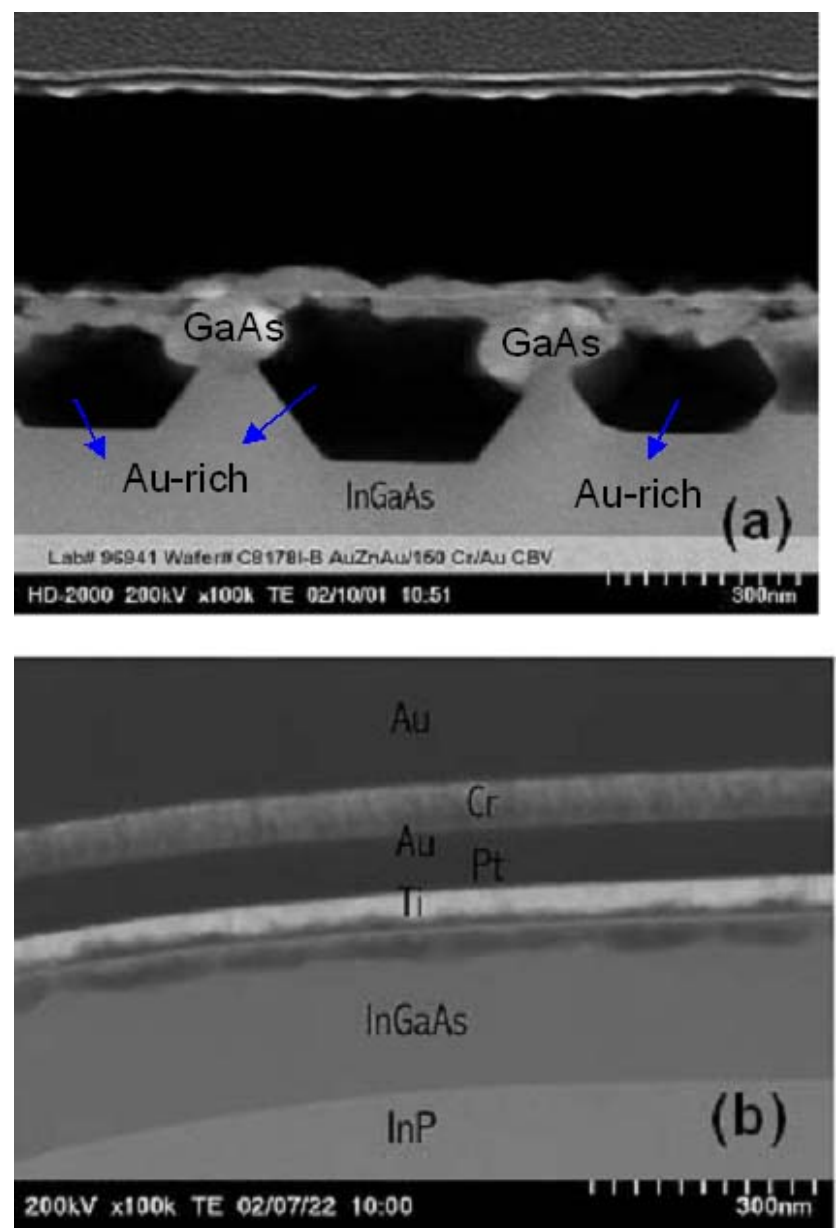

Figure 8. TEM images of the (a) $\mathrm{Au} / \mathrm{Zn} / \mathrm{Cr} / \mathrm{Au}$ and (b) $\mathrm{Au} / \mathrm{Ti} / \mathrm{Pt} / \mathrm{Au} / \mathrm{Cr} / \mathrm{Au}$ contacts

\subsubsection{Dielectric Uniformity}

In addition to the difference between the $\mathrm{Au} / \mathrm{Zn}$ and $\mathrm{Au} / \mathrm{Ti} / \mathrm{Pt} / \mathrm{Au}$, the dielectric conformity after the contact opening also played an important role. Figure 9 shows the cross-sectional SEM image of the degraded laser due to Au migration. The sample was prepared by focused ion beam (FIB). As shown by the FIB-SEM, there was no dielectric coverage at the upper left corner of the laser stripe resulting from overetch. At the corner, the Pt layer was likely not as uniform and conformal as that on the top. For the area where the Pt layer became thin or discontinuous, the effectiveness of the diffusion barrier decreased. During device operation, the weak spot eventually led to direct contact between $\mathrm{Au}$ and InP and caused massive Au diffusion into the InP area.

To explain the influence of contact process on defect formation and laser degradation, we have developed a defect formation and propagation model shown in Figure 10. After contact etch process by RIE, a surface damaged region was initially formed as marked by " $X$ ". During device operation and aging, the defects under the current driving force were propagating towards the active region. The defects that entered the active region may be in the form of point defects initially. The point defects may lead to nucleation and growth of dislocations over time, eventually forming dark spot defect (DSD) and/or dark line defect (DLD).

Figure 11(a) and (b) show the active region before and after the burn-in degradation, respectively. The active region before burn-in appeared intact with negligible defect density, while the DSD and DLD defects were formed at the active region after device degradation. The degradation at the active region led to threshold current increase and quantum efficiency decrease. 

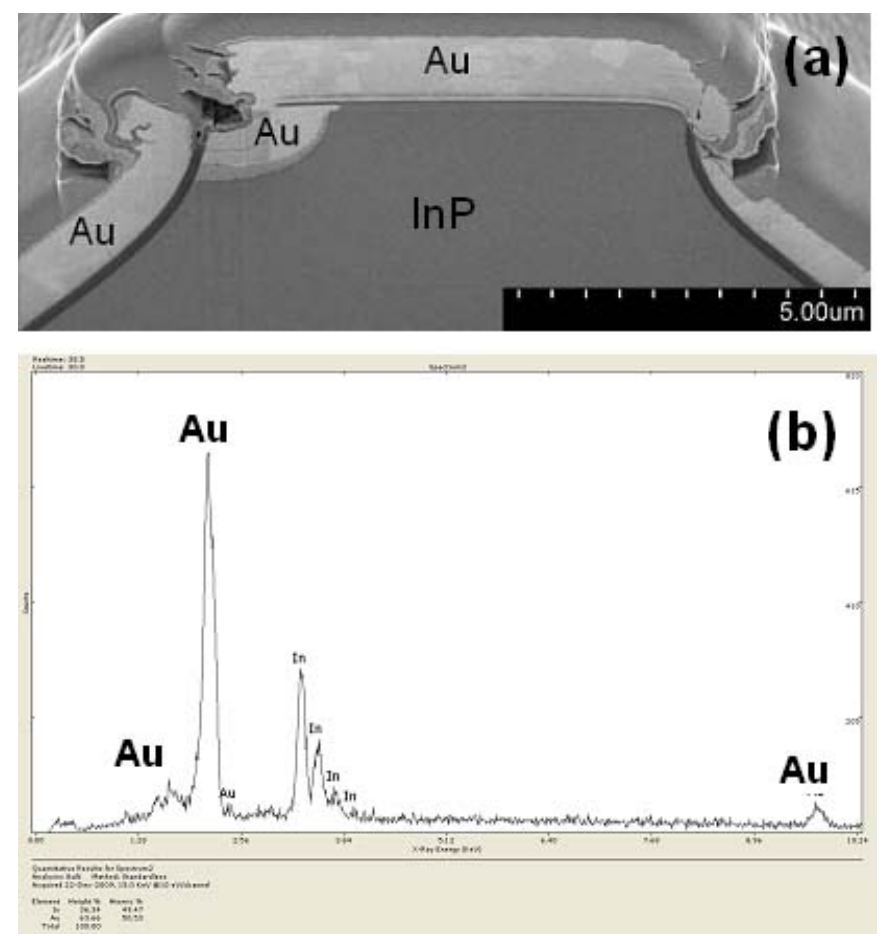

Figure 9. (a) FIB-SEM showing the Au migration from the contact to the active region. (b) Energy-dispersion spectroscopy (EDS) showing the Au peak

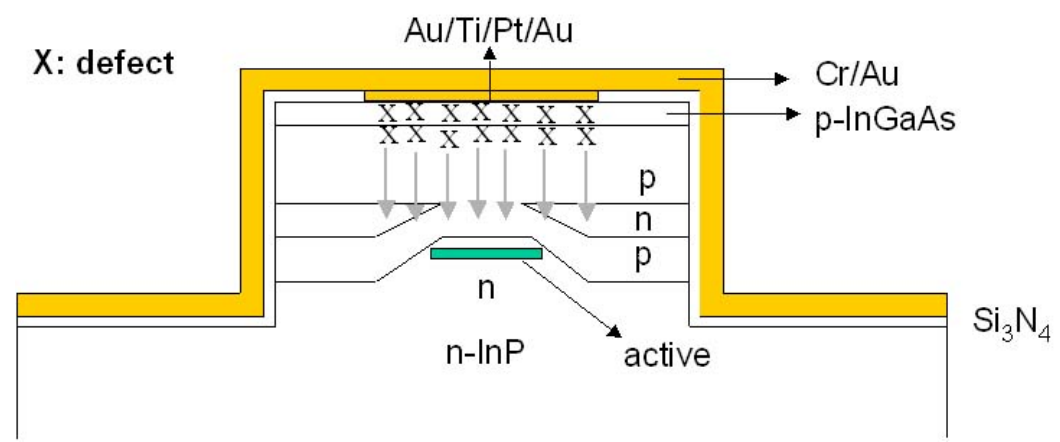

Figure 10. Schematic of defect formation and propagation model for BH laser

\subsection{Epitaxial Regrowth Interface}

Another major laser degradation mechanism was associated with the epi-regrowth interface defects (Fukuda, 1988). The quality of the BH interface between the etched mesa sidewall and burying layer was found to be critical in reliability performance. The reliability degradation was found to correlate with the residual damage at the sidewalls of the mesa (Kallstenius et al., 1999). The probable causes of the residual damage included inadequate removal of process-induced defects and defect generation during the epitaxial regrowth. For the latter, the III-V ratio and Zn-dopant were typically important factors. The common remedies included the addition of subsequent wet etch to refine the sidewall surface and the insertion of a buffer layer to prevent the $\mathrm{Zn}$ diffusion (Oohashi et al., 1998). For the former, defects could originate from the RIE-induced steps. The defects served as the non-radiative recombination sites that were responsible for laser degradation. 
(a)

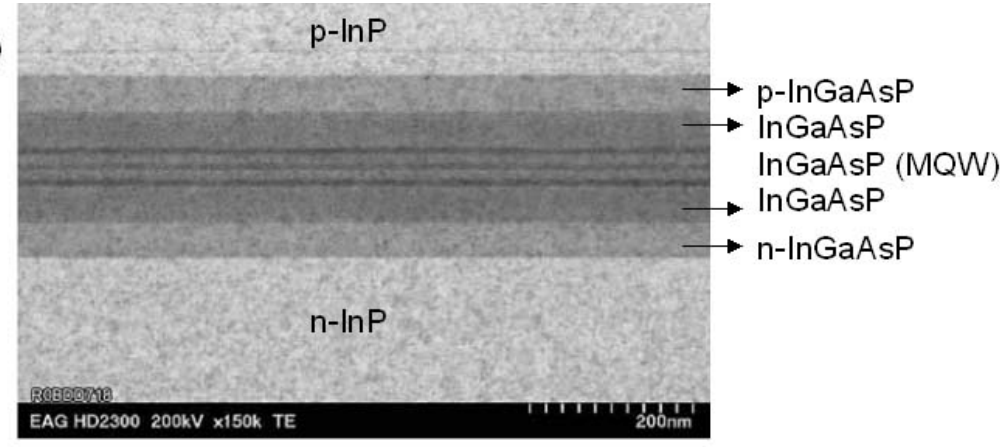

(b)

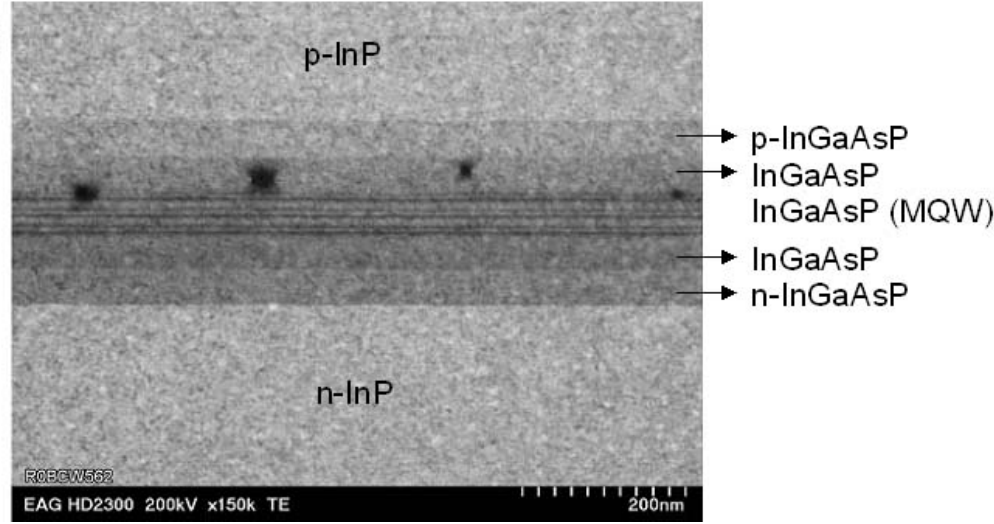

Figure 11. SEM image of the active region (a) before and (b) after burn-in degradation. The degraded laser showed DSD/DLD defect at the active region
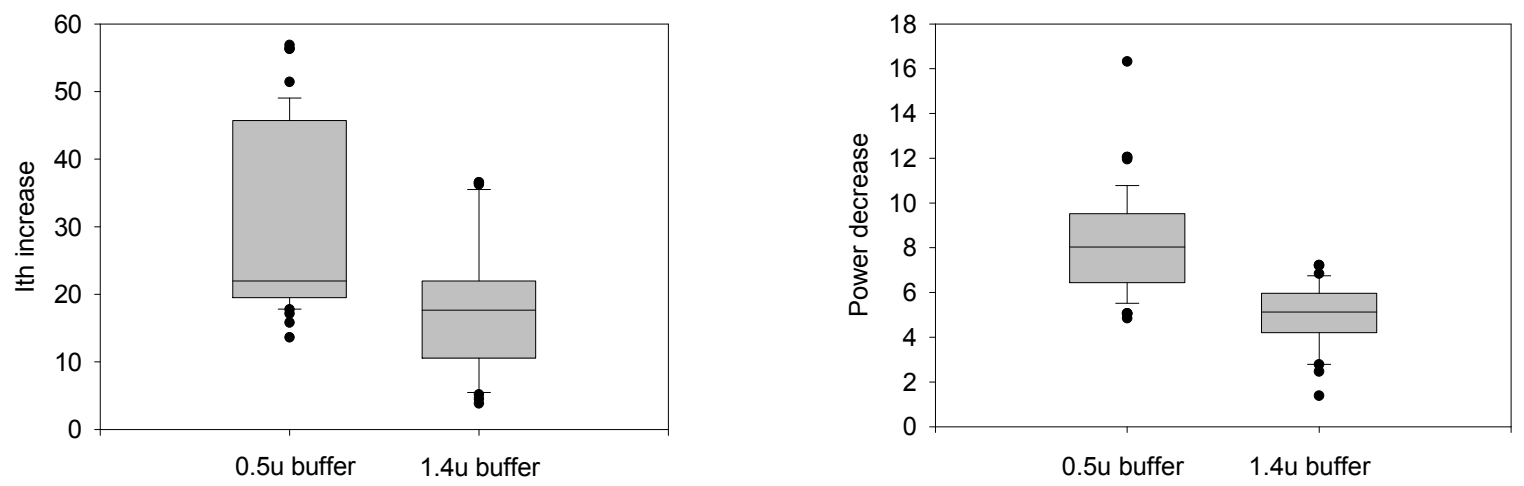

Figure 12. Life test comparison of $0.5 \mu \mathrm{m}$ vs. $1.4 \mu \mathrm{m}$ buffer layers (a) threshold current and (b) power changes

\subsection{Substrate Quality}

Reliability characteristics of wafers with different substrate vendors and buffer layers are summarized in Table I. The laser chips of 360 micrometer cavity were stressed with a constant stress current of $150 \mathrm{~mA}$ at $100{ }^{\circ} \mathrm{C}$. The p-contact of the lasers was $\mathrm{Au} / \mathrm{Zn}$. For 0.5 micrometer buffer, there appeared to be variation between wafers. The $\mathrm{I}_{\text {th }}$ change after 1000-hour aging was as high as $47 \%$. Such variation could be attributed to the quality of the substrate. For 1.4 micrometer buffer, the life test performance appeared to be more robust and consistent than the 0.5 micrometer buffer. For example, the Ith degradation from A-3 and A-4 was smaller than that from A-1 and A-2. Figures 12 (a) and (b) shows the comparison of threshold current and power changes between the two buffer layers. The 1000-hour life test data indicated that the incorporation of thicker buffer layer resulted in significant reliability improvement. The threshold current degradation after $1000 \mathrm{hr}$ was reduced from $22 \%$ to $16 \%$. The power degradation was reduced from $8 \%$ to $5 \%$. 
Table 1. Life test performance of wafers from different substrate vendors

\begin{tabular}{ccccc}
\hline Vendor & Buffer & Sample size & Ith(1000hr)/Ith(0) (\%) & Ith(1000hr)/Ith(0) (mA) \\
\hline A-1 & $0.5 \mu$ & 14 & 47 & 4.80 \\
A-2 & $0.5 \mu$ & 13 & 27 & 2.50 \\
B-1 & $0.5 \mu$ & 8 & 13 & 1.46 \\
B-2 & $0.5 \mu$ & 8 & 7 & 0.90 \\
C-1 & $0.5 \mu$ & 8 & 20 & 1.68 \\
C-2 & $0.5 \mu$ & 8 & 22 & 1.61 \\
A-3 & $1.4 \mu$ & 8 & 6 & 0.4 \\
A-4 & $1.4 \mu$ & 8 & 33 & 2.5 \\
C-3 & $1.4 \mu$ & 8 & 20 & 1.70 \\
C-4 & $1.4 \mu$ & 8 & 12 & 0.93 \\
\hline
\end{tabular}

To understand the reliability improvement in the thick buffer structure, the mesa etch and regrowth defect mechanism was proposed as shown in Figs. 13(a) and (b). In the thin buffer structure, the mesa etch punched through the substrate and the epi-regrowth was interacting with the defects in the substrate. The mesa etch/substrate interface became defective as marked by " $x$ " in Figure 13(a). During the aging, the defects were likely migrating toward the active region and degrade the lasing efficiency. In the thick buffer structure, there was no interaction between mesa etch and the substrate. Consequently, the active region was less susceptible to the defect formation during aging.

Alternatively, the reduction of laser lifetime in the thin buffer structure could be related to the growth and propagation of dislocation networks arising from the substrate crystal due to recombination enhanced defect motion (Fukuda, 2000). The defect motion could occur via climb and glide motion.

(a)

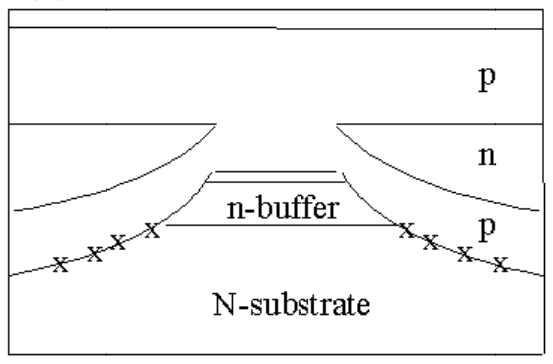

(b)

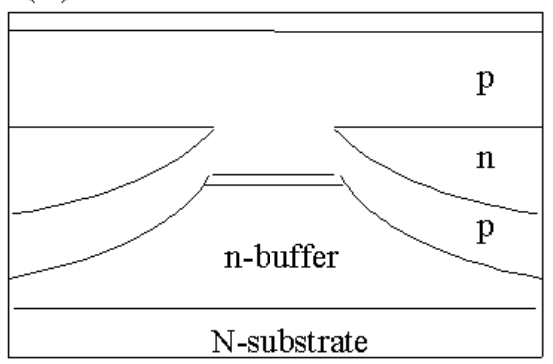

Figure 13. (a) BH laser with $0.5 \mu \mathrm{m}$ buffer layer (b) BH laser with $1.4 \mu \mathrm{m}$ buffer layer

\subsection{Laser Cavity Length}

Figure 14(a) shows the burn-in $I_{\text {th }}$ shift as a function of laser cavity length. Figure 14(b) shows the burn-in $I_{\text {th }}$ shift as a function of stress current density. The current density is defined as the stress current divided by the cavity length. The burn-in $\mathrm{I}_{\text {th }}$ shift seemed to show stronger dependence of the cavity length compared to the current density. For the cavity length dependence, the $\mathrm{I}_{\mathrm{th}}$ shift seemed to be the larger for the 1000 micrometer \& 1250 micrometer long lasers, which were attributable to the thin InGaAs layer and the higher number of defects in the long cavity lasers. It was also noted that other additional degradation mechanism such as mechanical stress could be introduced for longer cavity lasers. The mechanical stress caused by the mismatch of the thermal expansion coefficients between the InP laser chip and AlN submount became more important as the laser cavity increased (Westphalen, et al., 2009). The current densities of the 1000 micrometer \& 1250 micrometer lasers 
were among the lowest, suggesting that the current density was not the dominant effect. This was also supported by the short cavity data where the Ith shift of the 300 micrometer lasers was the lowest despite of the highest current density. The larger Ith change at the current density of $0.35 \mathrm{~mA} / \mathrm{micrometer}$ was attributed to the thin InGaAs. The test group of $0.35 \mathrm{~mA} /$ micrometer was based on 1000 and 1250 micrometer long lasers. The InGaAs contact layers of the lasers were about 0.1 micrometer, thinner than those of the other groups with the InGaAs thickness of 0.2 to 0.4 micrometer.

(a)

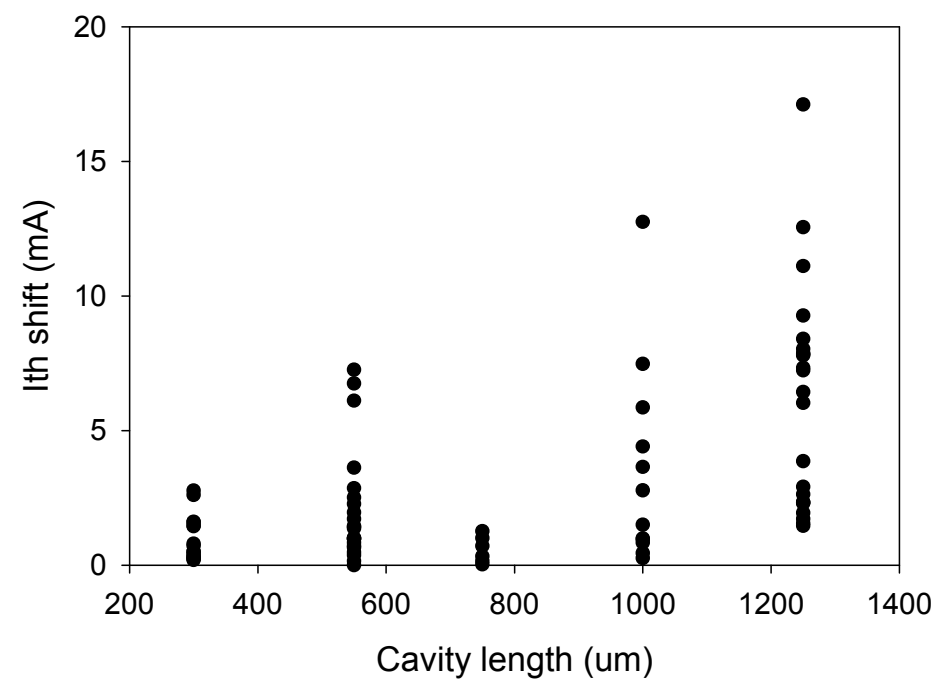

(b)

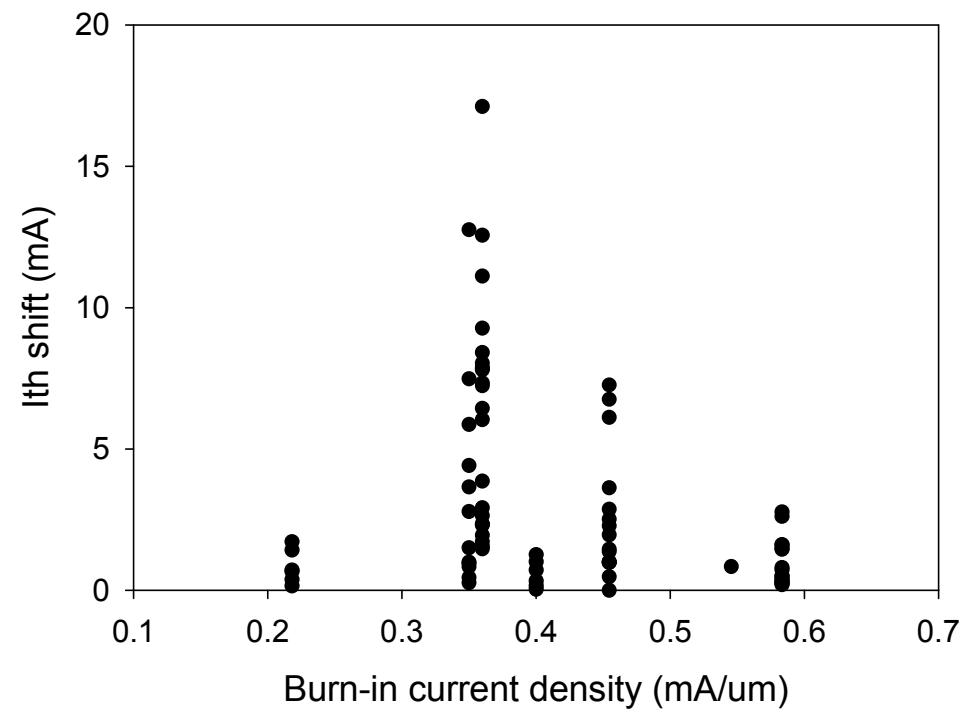

Figure 14. (a) The Ith changes of the lasers with different cavity lengths (b) The Ith changes as a function of current density. The current density was defined as the current divided by cavity length

\subsection{Robust Design-in Reliability Performance}

We have succeeded in suppressing laser degradation by means of optimization in contact opening process and regrowth. In the following, we will discuss the influential parameters of lasing wavelength and its correlation with laser reliability. We will show that the wavelength stability of the designed-in buried heterostructure lasers would meet the state-of-the-art stringent wavelength stability requirement.

The lasing mode of the DFB lasers is defined by the diffraction grating structure. The structure builds a one dimensional interference grating (or Bragg's grating), and the grating provides the optical feedback for the laser 
(A. Yariv, 1989). The lasing wavelength of the DFB laser is determined by the grating pitch and effective refractive index, as shown in Equation-1 where $\lambda_{\mathrm{DFB}}$ is the lasing wavelength, $\mathrm{n}_{\mathrm{eff}}$ is the effective refractive index, $\Lambda$ is the grating pitch and $\mathrm{m}$ is the order of grating.

$$
\lambda_{\text {DFB }}=2 \mathrm{n}_{\text {eff }} \Lambda / \mathrm{m}
$$

One primary cause for the wavelength shift is heating. As the temperature change occurs, the bandgap of the semiconductor laser and the thermal expansion change. As a result, the refractive index and the grating pitch show temperature dependence. The other factor is mechanical stress. The mechanical stress induced by the oxidation at the interface between the semiconductor and optical coating film was observed in $980 \mathrm{~nm}$ high power GaAs lasers (Martin-Martin et al., 2008; Landi et al., 1999). The stress at the laser facet is expected to be significantly less for the InP lasers due to the suppression of facet oxidation. The other type of mechanical stress is packaging stress induced by the die attach soldering process. Such packaging stress was created by the thermal expansion mismatch between the laser chip and the AlN heat sink submount. To isolate this factor, the solder, the soldering condition, and the submount used for the wavelength drift study were the same.

Experimentally, there are two common causes for wavelength drift of DFB lasers. One is related to the Ith increase during aging, and the other is related to heat generation. The former is widely observed in the constant power operation where the bias current is increased during device aging in order to maintain the same optical output power. The latter typically occurs in the constant current operation mode where the output power drop would lead to heat generation. The temperature rise would cause wavelength to increase (red shift).

In the following, we discuss the wavelength drift for the constant power operation mode. Table II shows examples of $\mathrm{I}_{\mathrm{th}}$ shift and resultant wavelength shift for both $1310 \mathrm{~nm}$ and $1550 \mathrm{~nm}$ DFB lasers. For $1310 \mathrm{~nm}$ lasers, the wavelength shift rate was measured to be about $0.0220 \mathrm{~nm} / \mathrm{mA}$. The $\mathrm{I}_{\mathrm{th}}$ shifts after $1000 \mathrm{hr}$ and 5000 $\mathrm{hr}$ life test aging were 0.40 and $0.47 \mathrm{~mA}$, respectively. As a result, the deduced wavelength shifts based on the Ith shift were estimated to be 0.0085 and $0.0103 \mathrm{~nm}$, respectively. It was shown that the magnitude of shift from laser degradation was small compared to the modern DWDM wavelength requirement of $0.03-0.09 \mathrm{~nm}$. For 1550 $\mathrm{nm}$ lasers, the wavelength shift rate was measured to be about $0.0065 \mathrm{~nm} / \mathrm{mA}$. The $\mathrm{I}_{\text {th }}$ shifts after $1000 \mathrm{hr}$ and $5000 \mathrm{hr}$ life test aging were 1.04 and $1.90 \mathrm{~mA}$, respectively. As a result, the deduced wavelength shifts based on the $\mathrm{I}_{\text {th }}$ shift were estimated to be 0.0068 and $0.0123 \mathrm{~nm}$, respectively. Again, the wavelength drift from intrinsic laser degradation was small compared to the modern DWDM wavelength requirement of 0.03-0.09 nm.

Table 2. Ith shift and WL drift data of $1310 \mathrm{~nm}$ and 1550nm DFB BH lasers

\begin{tabular}{ccccc}
\hline Aging & Cavity $(\mathrm{um})$ & $\mathrm{dWL} / \mathrm{dI}(\mathrm{nm} / \mathrm{mA})$ & Ith shift $(\mathrm{mA})$ & WL shift $(\mathrm{nm})$ \\
\hline $1000 \mathrm{hr}$ & 300 & 0.0220 & 0.40 & 0.0085 \\
$5000 \mathrm{hr}$ & 300 & 0.0220 & 0.47 & 0.0103 \\
$1000 \mathrm{hr}$ & 550 & 0.0065 & 1.04 & 0.0068 \\
$5000 \mathrm{hr}$ & 550 & 0.0065 & 1.90 & 0.0123 \\
\hline
\end{tabular}

\section{Conclusion}

We have discussed the key process parameters responsible for robust reliability performance. For p-contact effect, the contact etch, p-metal and dielectric uniformity were among the important factors. The defect formation and propagation model was developed to explain the effect of p-contact. For mesa etch interface, the wet etch and regrowth were found to be influential. For substrate quality, the thickness of buffer showed influence in laser degradation likely due to the defect formation between the substrate and epi-regrowth interface. The 1.4 micrometer thick buffer appeared to offer a good remedy in $\mathrm{I}_{\text {th }}$ change reduction.

The laser degradation showed stronger correlation with the laser cavity length than the current density. For example, the 300 micrometer long lasers showed the smallest Ith degradation despite of the highest current density.

\section{Acknowlegments}

The author would like to thank Chun Lei and Harqkyun Kim at Emcore (Alhambra, CA) for helpful discussions, Ruby Zendejaz at Emcore (Alhambra, CA) for assistance in wafer processing and Jane Martin at Evan Analytical Group (North Carolina) for assistance in TEM, STEM and EDS. 


\section{References}

Amnon, Yariv. (1989). Quantum Electronics. New York: Wiley.

Cheung, K. P., \& Chang, C. P. (1994). Plasma-charging damage: A physical model. J. Appl. Phys., 75, 4415-4426. http://dx.doi.org/10.1063/1.355985

Chin, A. K., Zipfel, C. L., Geva, Gamlibel, M. I., Skeath, D., \& Chin, B. K. (1984). Direct evidence for the role of gold migration in the formation of dark-spot defects in $1.3 \mathrm{um} \mathrm{InP/InGaAsP}$ light-emitting diodes. Appl. Phys. Lett., 45, 37-39. http://dx.doi.org/10.1063/1.94995

Dylewicz, R., De La Rue, R. M., Wasielewski, R., Mazur, P., Mezosi, G., \& Bryce, A. C. (2010). Fabrication of submicron-sized features in $\mathrm{InP} / \mathrm{InGaAsP} / \mathrm{AlGaInAs}$ quantum well heterostructures by optimized inductively coupled plasma etching with Cl2/Ar/N2 chemistry. J. Vacuum Sci. Tech. B, 28, 882-890. http://dx.doi.org/10.1116/1.3466811

Foad, M. A., Thoms, S., \& Wilkinson, C. D. W. (1993). New technique for dry etch damage assessment of semiconductors. J. Vac. Sci. Tech. B, 11, 20-25. http://dx.doi.org/10.1116/1.586720

Fukuda, M. (1988). Laser and LED reliability update. J. Lightwave Tech., 6, 1488-1495. http://dx.doi.org/10.1109/50.7906

Fukuda, M. (2000). Historical overview and future of optoelectronics reliability for optical fiber communication systems. Microelectronics Reliab., 40, 27-35. http://dx.doi.org/10.1016/S0026-2714(99)00236-X

Fukuda, M., Noguchi, Y., Motosugi, G., Nakano, Y., Tsuzuki, N., \& Fujita, O. (1987). Suppression of interface degradation in InGaAsP/InP buried heterostructure lasers. J. Lightwave Tech., LT-5, 1778-1781. http://dx.doi.org/10.1109/JLT.1987.1075475

Fukuda, M., Wakita, K., \& Iwane, G. (1983). Dark defects in InGaAsP/InP double heterostructure lasers under accelerated aging. J. Appl. Phys., 1246-1250. http://dx.doi.org/10.1063/1.332186

Giannuzzi, L. A., \& Stevie, F. A. (1999). A review of focused ion beam milling techniques for TEM specimen preparation, Micron, 30, 197-204. http://dx.doi.org/10.1016/S0968-4328(99)00005-0

Huang, J. S. (2005). Temperature and current dependencies of reliability degradation of buried heterostructure semiconductor lasers. IEEE Trans. Device Mater. Reliab., 5, 150-154. http://dx.doi.org/10.1109/TDMR.2005.843834

Huang, J. S. (2011). Design-in reliability of modern distributed feedback (DFB) InP lasers: Can we meet up the stringent wavelength-division multiplex (WDM) requirement? IEEE Photonic Society Summer Topical (July 18-20, 2011, Montreal, Quebec, Canada), 89-90.

Huang, J. S., \& Vartuli, C. B. (1988). The effect of Cr barrier on interfacial reaction of $\mathrm{Au} / \mathrm{Zn} / \mathrm{Au} / \mathrm{Cr} / \mathrm{Au}$ contacts to p-type InGaAs/InP. Thin Solid Films, 446, 132-137. http://dx.doi.org/10.1016/S0040-6090(03)01345-2

Huang, J. S., \& Vartuli, C. B. (2003). Scanning transmission electron microscopy study of $\mathrm{Au} / \mathrm{Zn} / \mathrm{Au} / \mathrm{Cr} / \mathrm{Au}$ and $\mathrm{Au} / \mathrm{Ti} / \mathrm{Pt} / \mathrm{Au} / \mathrm{Cr} / \mathrm{Au}$ contacts to p-type InGaAs/InP. J. Appl. Phys., 93, 5196-5200. http://dx.doi.org/10.1063/1.1565187

Huang, J. S., Chen, C., Yeh, C. C., Tu, K. N., Shofner, T. L., Drown, J. L., Irwin, R. B., \& Vartuli, C. B. (2000).

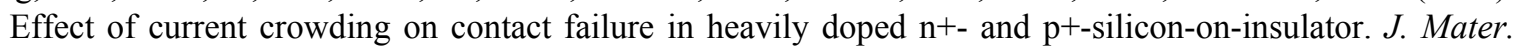
Res., 15, 2387-2392. http://dx.doi.org/10.1557/JMR.2000.0343

Huang, J. S., Miao, R., Lu, H., Wang, C., Helms, C., \& Demirci, H. (2007). Theoretical and experimental thermal analysis of InP ridge lasers on submounts and TO packages. IEEE Trans. Device Mater. Reliab., 7 , 363-368. http://dx.doi.org/10.1109/TDMR.2007.901637

Huang, J. S., Nguyen, T., Hsin, W., Aeby, I., Ceballo, R., \& Krogen, J. (2005). Reliability of etched-mesa buried heterostructure semiconductor lasers. IEEE Trans. Device Mater. Reliab., 5, 665-674. http://dx.doi.org/10.1109/TDMR.2005.860562

Huang, J. S., Shofner, T. L., \& Zhao, J. (2000). Direct observation of void morphology in step-like electromigration resistance behavior and its correlation with critical current density. J. Appl. Phys., 89, 2130-2133. http://dx.doi.org/10.1063/1.1340004

Ishino, M., Takenaka, N., Kito, M., Fujihara, K., Ostuka, N., Fujito, K., \& Matsui, Y. (1997). Low distortion $1.3 \mathrm{~m}$ strained-layer MQW-DFB laser for AM-SCM transmission systems with large channel capacity. $J$. Lightwave Tech., 15(11), 2172-2178, 1997. http://dx.doi.org/10.1109/50.641539 
Itoh, M., Kondo, Y., Kishi, K., Yamamoto, M., \& Itaya, Y. (1996). High-quality 1.3um GaInGaP-BH-lasers fabricated by MOVPE and dry-etching technique. IEEE Photon Tech. Lett., 8, 989-991. http://dx.doi.org/10.1109/68.508713

Kallstenius, T., Backstrom, J., Smith, U., \& Stoltz, B. (1999). Degradation of InGaAsP/InP-based multiquantum-well lasers. J. Appl. Phys., 86, 2397-2406. http://dx.doi.org/10.1063/1.371067

Kallstenius, T., Backstrom, J., Smith, U., \& Stoltz, B. (1999). On the degradation of InGaAsP/InP-based bulk lasers. J. Lightwave Tech., 17, 2397-2406. http://dx.doi.org/10.1109/50.809681

Landi, S., Papuzza, C., Piccirillo, A., \& Serra, D. R. L. (1999). Characterization of III-V optoelectronic devices by internal second-harmonic generation technique. Appl. Phys. Sci., 143, 115-123.

Martin-Martin, A., Avella, M., Iniguez, M. P., Jimenez, J., Oudart, M., \& Nagle, J. (2008). A physical model for the rapid degradation of semiconductor laser diodes. Appl. Phys. Lett., 93, 171106. http://dx.doi.org/10.1063/1.3009290

Mizuishi, K., Sawai, M. Todoroki, S. Tsuji, S. Hirao, M., \& Nakamura, M. (1983). Reliability of InGaInP/InP buried heterostructure 1.3um lasers. IEEE J. Quantum Electron. QE-19, 1294-1301. http://dx.doi.org/10.1109/JQE.1983.1072019

Morello, G., Quaglic, M., Meneghini, G., Papuzza, C., \& Kompocholis, C. (2006). Reactive ion etching induced damage evaluation for optoelectronic device fabrication. J. Vac. Sci. Tech. B, 24, 756-761. http://dx.doi.org/10.1116/1.2181576

Oohashi, H., Fukuda, M., Kondo, Y., Wada, M., Tohmori, Y., Sakai, Y., Toba, H., \& Itaya, Y. (1998). Reliability of 1300-nm spot-size converter integrated laser diodes for low-cost optical modules in access networks. J. Lightwave Tech., 16, 1302-1307. http://dx.doi.org/10.1109/50.701409

Pearton, S. J., Ren, F., Abernathy, C. R., Hobson, W. S., Fullowan, T. R., Esagui, R., \& Lothian, J. R. (1992). Damage introduction in InP and InGaAs during Ar and H2 plasma exposure. Appl. Phys. Lett., 61, 586-588. http://dx.doi.org/10.1063/1.107845

Westphalen, T., Leers, M., Werner, M., Traub, M., Hoffmann, H. D., \& Ostendorf, R. (2009). Packaging influence on laser bars of different dimensions. Proc. SPIE, 7198, K-1.

Yang, S. H., \& Bandaru, P. R. (2007). An experimental study of the reactive ion etching (RIE) of GaP using BCl3 plasma processing. Mater. Sci. Eng. B-Solid State Mater. Adv. Tech., 143, 27-30. 